\title{
Taste-related reward is associated with weight loss following bariatric surgery
}

\author{
Kimberly R. Smith, ${ }^{1}$ Afroditi Papantoni, ${ }^{2}$ Maria G. Veldhuizen, ${ }^{3}$ Vidyulata Kamath,${ }^{4}$ Civonnia Harris, ${ }^{5}$ Timothy H. Moran, ${ }^{1}$ \\ Susan Carnell, ${ }^{2}$ and Kimberley E. Steele ${ }^{5,6}$ \\ 'Department of Psychiatry \& Behavioral Sciences, and 2Division of Child and Adolescent Psychiatry, Department of Psychiatry \& Behavioral Sciences, Johns Hopkins University School of Medicine, \\ Baltimore, Maryland, USA. ${ }^{3}$ Anatomy Department, Mersin University School of Medicine, Mersin, Turkey. ${ }^{4}$ Division of Medical Psychology, Department of Psychiatry \& Behavioral Sciences, Johns Hopkins \\ University School of Medicine, Baltimore, Maryland, USA. ${ }^{5}$ Department of Surgery, Johns Hopkins Center for Bariatric Surgery, Johns Hopkins University School of Medicine, Baltimore, Maryland, USA. \\ ${ }^{6}$ Department of Health, Behavior and Society, Johns Hopkins Bloomberg School of Public Health, Baltimore, Maryland, USA.
}

\begin{abstract}
BACKGROUND. Bariatric surgeries are the most effective treatments for successful and sustained weight loss, but individuals vary in treatment response. Understanding the neurobiological and behavioral mechanisms accounting for this variation could lead to the development of personalized therapeutic approaches and improve treatment outcomes. The primary objectives of this study were to investigate changes in taste preferences and taste-induced brain responses after Roux-en- $Y$ gastric bypass (RYCB) and vertical sleeve gastrectomy (VSC) and to identify potential taste-related predictors of weight loss.
\end{abstract}

METHODS. Females, ages 18 to 55, with a body mass index greater than or equal to $35 \mathrm{~kg} / \mathrm{m}^{2}$, and approved for bariatric surgery at the Johns Hopkins Center for Bariatric Surgery were recruited for participation. Demographics, anthropometrics, liking ratings, and neural responses to varying concentrations of sucrose plus fat mixtures were assessed before and after surgery via visual analog scales and functional MRI.

RESULTS. Bariatric surgery produced decreases in liking for sucrose-sweetened mixtures. Greater preference for sucrosesweetened mixtures before surgery was associated with greater weight loss in RYGB, but not VSC. In the RYGB group only, individuals who showed lower taste-induced activation in the ventral tegmental area (VTA) before surgery and greater changes in taste-induced VTA activation 2 weeks following surgery experienced increased weight loss.

CONCLUSION. The anatomical and/or metabolic changes associated with RYCB may more effectively "reset" the neural processing of reward stimuli, thereby rescuing the blunted activation in the mesolimbic pathway found in patients with obesity. Further, these findings suggest that RYGB may be particularly effective in patients with a preference for sweet foods.

FUNDING. NIH K23DK100559 and Dalio Philanthropies.

\section{Introduction}

Bariatric surgery results in substantial and sustained weight loss, making it the most successful treatment to date for obesity (1-3). The 2 most common bariatric interventions are the Roux-en-Y gastric bypass (RYGB) and the vertical sleeve gastrectomy (VSG). In both surgeries, the surgeon physically alters the normal integrity of the gastrointestinal tract, reducing the stomach to a fraction of its original size, from approximately 80 to $120 \mathrm{~mL}$ in VSG and approximately 20 to $30 \mathrm{~mL}$ in RYGB. Additionally, RYGB involves transection of the proximal jejunum, attachment of the Roux limb to the gastric pouch, and reattachment of the stomach, duodenum, and proximal jejunum to the distal jejunum. Changes in various mechanisms involved in the homeostatic and hedonic control of food intake have been suggested as contributing to

Conflict of interest: The authors have declared that no conflict of interest exists. Copyright: () 2020, American Society for Clinical Investigation.

Submitted: March 4, 2020; Accepted: May 13, 2020; Published: July 13, 2020.

Reference information: / Clin Invest. 2020;130(8):4370-4381.

https://doi.org/10.1172/JCI137772. the effectiveness of these bariatric interventions (see ref. 4 for review). Potential gastrointestinal mechanisms include reduced gastric capacity and removal or reduction of the ghrelin-producing cells of the stomach, increased postprandial anorexigenic gut peptide release, and alteration of the gut microbiome (e.g., refs. 5-12). However, our understanding of the impact of bariatric surgery on the central nervous system, more specifically on brain reward pathways, is limited (13).

The hedonic control of food intake is putatively mediated by the brain's opioid and dopamine systems, including the ventral tegmental area (VTA), striatum, and hypothalamus (see ref. 14 for review). Bariatric surgery appears to improve obesity-associated functional changes in central reward circuits (15). Previously, we reported that individuals with obesity who underwent RYGB had increases in striatal dopamine D2 receptor availability by PET (16). However, the directionality of these changes as assessed by PET is not universally observed (17). Other investigators have reported that reward circuit responses to food cues, as assessed by functional MRI (fMRI), are reduced (18-20). Psychophysical methods ranging in rigor have shown increases in sensitivity 
Table 1. Description and statistical outcomes of baseline participant demographics

$\begin{array}{lccc}\text { Demographics } & \text { VSC }(\boldsymbol{n}=25) & \text { RYGB }(\boldsymbol{n}=23) & \text { Statistical result } \\ \text { Age } & 38.92 \pm 1.51 & 40.0 \pm 1.92 & t_{46}=0.445, P=0.659 \\ \text { Race } & \text { W (9), AA (15), A (1), H (0) } & \text { W (16), AA (6), A (0), H (1) } & x_{3}^{2}=7.747, P=0.052 \\ \text { Weight } & 269.26 \pm 6.14 & 270.15 \pm 7.25 & t_{46}=0.094, P=0.926 \\ \text { BMI } & 43.41 \pm 0.99 & 44.56 \pm 1.06 & t_{46}=0.791, P=0.433 \\ \text { Preoperative comorbidities } & & & \\ \text { Type 2 diabetes mellitus } & 5(20.0 \%) & 5(21.7 \%) & x_{1}^{2}=0.022, P=0.882 \\ \text { Hypertension } & 11(44 \%) & 13(56.5 \%) & x_{1}^{2}=0.751, P=0.386 \\ \text { CERD } & 9(64 \%) & 18(78.3 \%) & x_{1}^{2}=8.694, P=0.003 \\ \text { Sleep apnea } & 12(48 \%) & 12(52.2 \%) & x_{1}^{2}=0.083, P=0.773 \\ \text { Hyperlipidemia } & 5(20 \%) & 4(17.4 \%) & x_{1}^{2}=0.054, P=0.817\end{array}$

Mean \pm SEM of participant age and preoperative/baseline body weight and BMI. Sample size $(n)$ of race in each group is shown. Sample size and percentage (\%) of participants with type 2 diabetes mellitus, hypertension, GERD, sleep apnea, and hyperlipidemia at baseline assessment are shown. W, white; $A A$, African American; A, Asian; H, Hispanic.

to (refs. 21-23; but see ref. 24) and decreases in palatability for (refs. 21, 24; but see refs. 22, 25) sweet-tasting foods and liquids in patients who received bariatric surgery. This is further supported by dietary recall measures and patient interviews that suggest a decrease in the selection and consumption of sugary foods following surgery (26-30), although such reports are variable $(24,31,32)$. Direct measurement of food intake via an ad libitum buffet meal showed no change in food preference 6 months following surgery (33). Whether RYGB and VSG yield similar behavioral and neural changes in hedonic processing is unknown. Furthermore, the time course for the development of surgery-induced neural and behavioral alterations is yet to be elucidated.

Our overarching hypothesis is that the robust weight loss observed following bariatric surgery results, in part, from neurobiologic changes in reward regions of the brain, specifically involvement of the mesolimbic dopaminergic pathway. Motivated by clinical observation and personal accounts from our bariatric surgical cohort, we hypothesized that bariatric surgery may alter taste preferences and thereby induce weight loss. Our primary aims were (a) to investigate bariatric surgery-induced changes in taste preference for sucrose- and fat-containing mixtures, as measured by taste-preference ratings using visual analog scales (VAS) and neural activation via blood oxygen level-dependent (BOLD) signaling before and after bariatric surgery, and (b) to evaluate associations of these measures with the degree of weight loss 6 months following surgery. We hypothesized that bariatric surgery would decrease liking for mixtures with high-sucrose or high-fat content and enhance neural responses to "sweet" and "fat" in regions implicated in taste and reward. Further, based on published patient reports (34), clinical observation, and direct personal accounts from the bariatric patients themselves, we expected these changes in taste preference to occur immediately following surgery and diminish over time, with more robust changes observed in patients who received RYGB relative to those receiving VSG. We also tested to determine whether baseline and shortterm changes in these behavioral (taste preference) and neural (BOLD signaling) measures would be associated with 6-month weight loss outcomes.

\section{Results}

\section{Participant demographics}

Among the 48 study participants (VSG: $n=25$, RYGB: $n=23$ ), all were female, and the baseline characteristics were similar between surgery groups, including age (VSG: $38.92 \pm 1.51$ vs. RYGB: $40.0 \pm 1.92$ ), race (VSG: $40 \%$ white vs. RYGB: $70 \%$ white), and baseline weight measures (BMI [VSG: $43.41 \pm 0.99$ lb vs. RYGB: $44.56 \pm 1.06 \mathrm{lb}$ ] and body weight [VSG: $269.26 \pm 6.14 \mathrm{lb}$ vs. RYGB: 270.15 $\pm 7.25 \mathrm{lb}]$ ). Groups were similar across obesity-related comorbidities, with the exception of gastroesophageal reflux disease (GERD) at baseline (VSG: 64\% vs. RYGB: $78.3 \%, P=0.003$ ). Complete participant demographics and statistical comparisons are listed in Table 1, with participant recruitment, enrollment, and participation depicted in Figure 1.

\section{Effectiveness of bariatric surgery on weight loss}

Bariatric surgery significantly reduced body weight in patients as early as 2 weeks, which was sustained to 6 months following surgery $(P<0.001)$. RYGB resulted in greater percentage of total weight loss (\%TWL; Figure 2A) relative to VSG at 3 months (\%TWL: $t_{46}$ $=2.421, P=0.019)$ and 6 months (\%TWL: $\left.t_{46}=2.988, P=0.004\right)$ following surgery, although \%TWL at 3 months did not withstand correction for multiple comparisons (corrected $P=0.057)$. Supplemental Figure 1 (supplemental material available online with this article; https://doi.org/10.1172/JCI137772DS1) presents data as changes in BMI and percentage of excess weight loss (\%EWL), and Supplemental Table 1 presents data expressed as changes in body weight, BMI, \%TWL, and \%EWL. For the remainder of the manuscript, \%TWL will be used to describe the associations of weight loss with behavioral and neuroimaging measures.

\section{Fasting hunger ratings}

There was no difference in preoperative fasting hunger ratings between the surgical groups $\left(t_{38}=-0.570, P=0.572\right)$. Bariatric surgery reduced hunger at 2 weeks after surgery (RYGB: $t_{19}=4.003$, $P=0.001$; VSG: $\left.t_{19}=2.991, P=0.008\right)$, but this change in hunger returned to baseline levels by 3 months (RYGB: $P=0.371$, VSG: $P=0.345$; see Supplemental Figure 2).

\section{Preoperative behavioral (taste preference) associations with weight loss}

Liking for sucrose-sweetened mixtures before surgery is associated with greater weight loss following RYGB, but not VSG. Preoperative liking ratings for mixtures with added sucrose were positively associated with 6-month \%TWL following RYGB, but not VSG (Figure 2B). Individuals who gave higher ratings to sugar-sweetened mixtures (skim milk with $20 \%$ added sucrose [ $P=0.041]$, half-and-half with $10 \%$ added sucrose $[P=0.014]$, and whole milk with $20 \%$ added sucrose $[P=0.004]$ ) before RYGB experienced greater \%TWL than those who gave lower ratings to the same mixtures. There was 


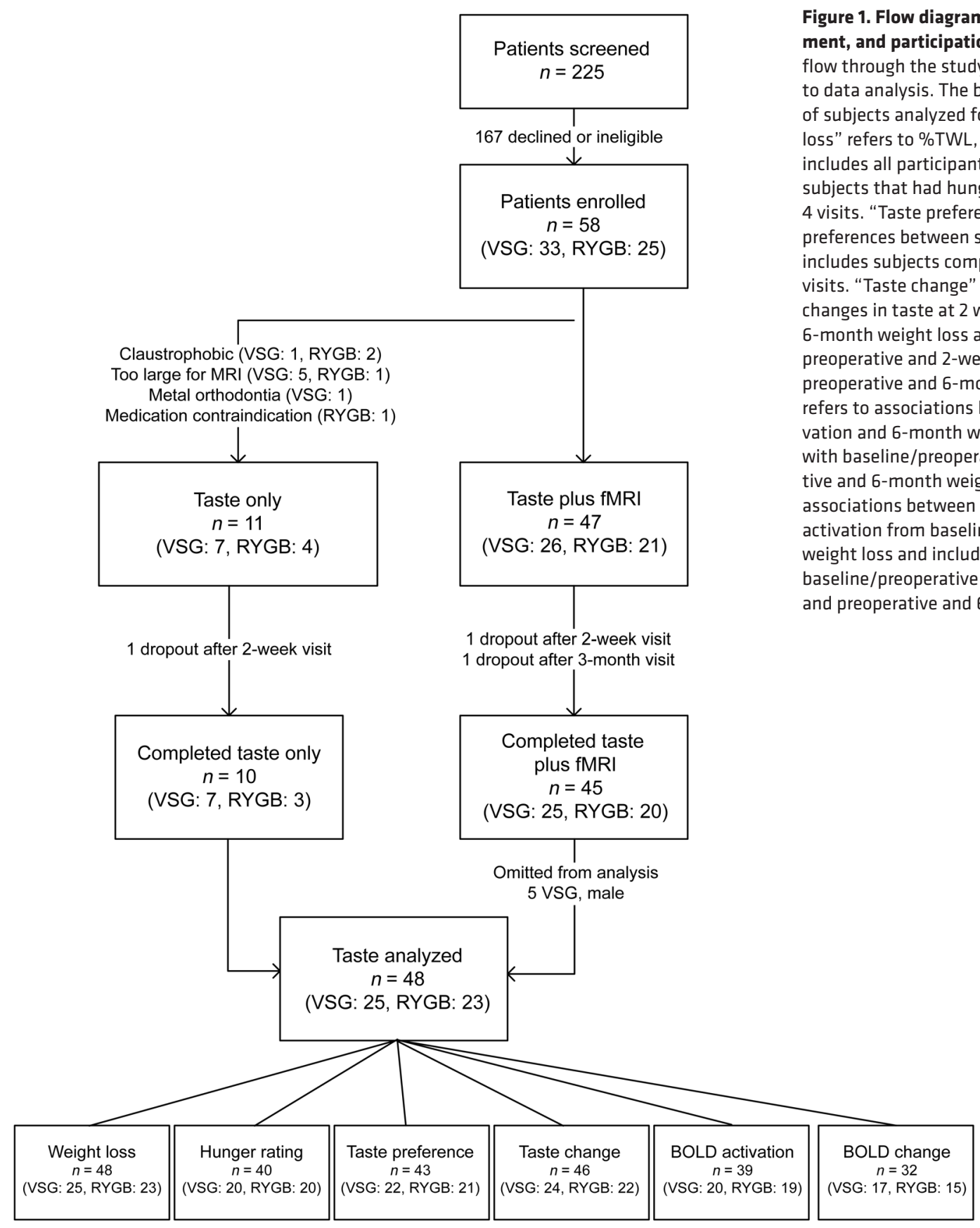

no relationship between preoperative ratings for sucrose-sweetened mixtures and \%TWL in the VSG group.

We further explored preoperative taste preference as a potential surgery-specific predictor of optimal weight loss. Bariatric patients were subdivided into 4 groups based on surgery type and preoperative ratings of each taste mixture (median split of scale 0-50 and 51-100 on VAS from 0-100). Patients with a higher liking for half-and-half with $10 \%$ added sucrose before surgery (preferrers) experienced significantly greater 6-month \% TWL following RYGB compared with patients who underwent RYGB and reported lower liking (nonpreferrers; $P=0.003$ ) or VSG patients reporting higher (preferrers; $P<0.001)$ or lower liking (nonpreferrers; $P=0.004$ ) (Figure $2 \mathrm{C}$ ).

\section{Postoperative behavioral (taste preference) associations} with weight loss

Changes in liking ratings for sucrose-containing mixtures. Before surgery, both groups demonstrated concentration-dependent liking for sucrose, with higher liking for the mixtures with added sucrose compared with no added sucrose (Supplemental Figure 3A). The intraclass correlation (ICC) value for preoperative liking ratings of the 8 sucrose-sweetened stimuli was $r=0.822$, indicating a high degree of reliability across ratings for the sucrose-sweetened mixtures. Supplemental Table 2 details the ICC for ratings for mixtures of each sucrose content. After surgery, liking ratings for mixtures with added sugar decreased significantly from baseline across both groups (Figure 3A). Two weeks following surgery, 
A

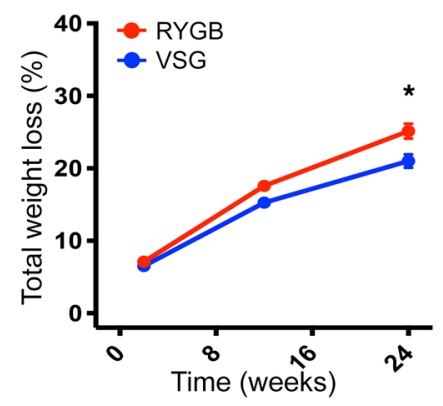

B Skim milk, 20\% added sucrose
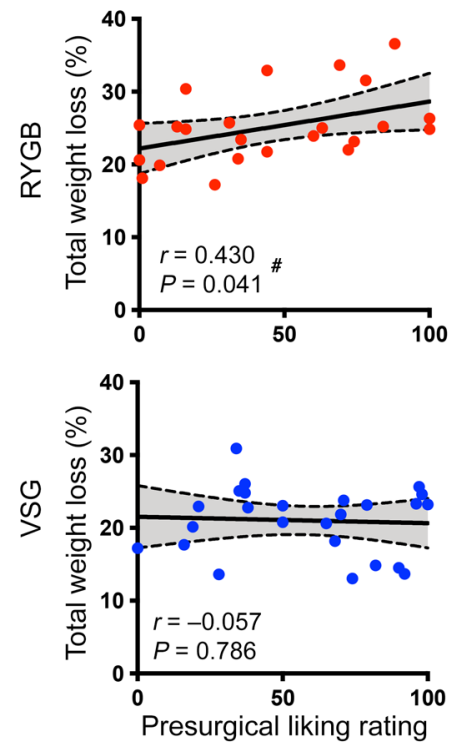

C

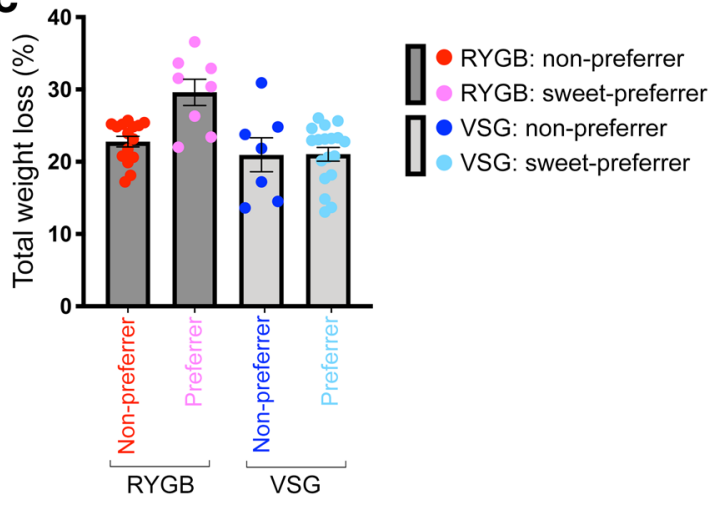

Whole milk, $20 \%$ added sucrose

Half \& Half, $10 \%$ added sucrose
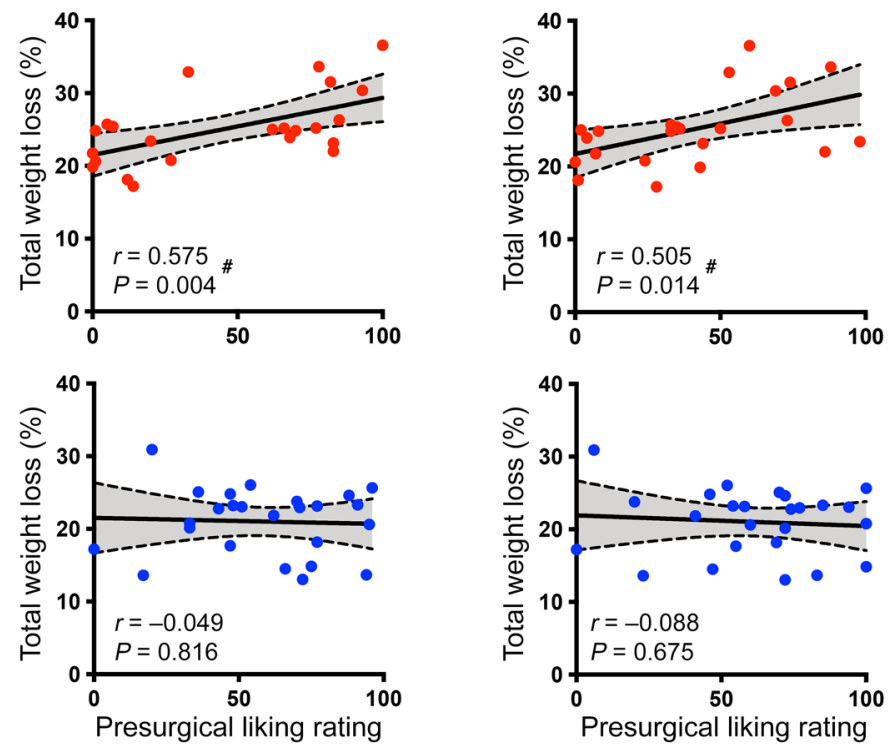

Figure 2. Preoperative liking of sucrose-sweetened taste mixtures predicts 6-month weight loss following RYGB. Weight loss and the relationship between preoperative liking ratings for sucrose+fat-containing mixtures and changes in weight at 6 months following RYGB and VSG. (A) Mean \pm SEM $\%$ TWL from preoperative/baseline ( 0 weeks) and 2 weeks, 3 months, and 6 months after surgery in RYGB (red; $n=23$ ) and VSG (blue; $n=25$ ) patients. Repeated-measures ANOVA revealed a main effect of time $\left(F_{14.63 .2}=513.882, P<0.001\right)$, group $\left(F_{146}=7.956, P=0.007\right)$, and time $\times$ group interaction $\left(F_{14.63 .2}\right.$ $=6.024, P=0.010$ ) for \%TWL. ${ }^{*} P<0.05$. (B) Preoperative ratings for mixtures with added sucrose correlated with \%TWL at 6 months following RYCB (red), but not following VSG (blue). "Significant bivariate correlations. (C) Mean \pm SEM \%TWL at 6 months following bariatric surgery in patients who gave a "liking" rating from 0 to 100 to half-and-half with $10 \%$ added sucrose. One-way ANOVA $\left(F_{3,44}=8.093, P<0.001\right)$ with Bonferroni's correction revealed greater \%TWL in RYGB preferrers relative to RYGB nonpreferrers $(P=0.004)$, VSG preferrers $(P<0.001)$, and VSG nonpreferrers $(P=0.002)$. \%TWL did not differ between RYGB nonpreferrers, VSG preferrers, and VSC nonpreferrers $(P=1.00)$. Red, 0-50 VAS liking rating, nonpreferrer, RYGB $(n=15)$; pink, 51-100 VAS liking rating, preferrer, RYGB $(n=8)$; blue, 0-50 VAS liking rating, nonpreferrer, VSG $(n=7)$; cyan, 51-100 VAS liking rating, preferrer, VSG ( $n=18)$.

concentration-dependent liking for sucrose was still apparent in both groups. However, by 3 months and at 6 months after RYGB, no differences in ratings for the $0 \%-20 \%$ added sugar mixtures were observed (Supplemental Figure 3A) due to decreased ratings for the mixtures with $10 \%$ and $20 \%$ added sucrose (Figure $3 \mathrm{~A}$ ). VSG had no effect on concentration-dependent sucrose liking (Supplemental Figure 3A).

Changes in liking ratings for fat-containing mixtures. No concentration-dependent liking for fat was observed for either surgical group before surgery (Supplemental Figure 3B). The ICC value for preoperative liking ratings of the 9 fat-containing stimuli was $r=0.598$, indicating moderate reliability across ratings for the mixtures with various levels of fat content. Supplemental Table 2 details the ICC for ratings for mixtures of each level of fat con- tent. At 2 weeks following surgery, both groups showed concentration-dependent liking for fat (RYGB: $P=0.030$, VSG: $P=0.002$; Supplemental Figure 3B), but this persisted only in the VSG group out to 3 months after surgery (RYGB: $P=0.731$, VSG: $P<0.001$; Supplemental Figure 3B). By 6 months, as observed preoperatively, concentration-dependent liking for fat was no longer present in either group ( $P=0.115$; Supplemental Figure $3 \mathrm{~B})$. Significant decreases in liking for whole milk were observed following RYGB, with the rating difference at 3 months from baseline surviving correction $\left(t_{20}=2.785, P=0.011\right.$, corrected $P=0.033$; Figure $\left.3 \mathrm{~B}\right)$. Liking for half-and-half was lower at baseline and 2 weeks and 3 months after surgery in RYGB compared with VSG recipients, with the group difference at 2 weeks surviving correction $\left(t_{41}=-3.041\right.$, $P=0.004$, corrected $P=0.016$; Figure 3B). 

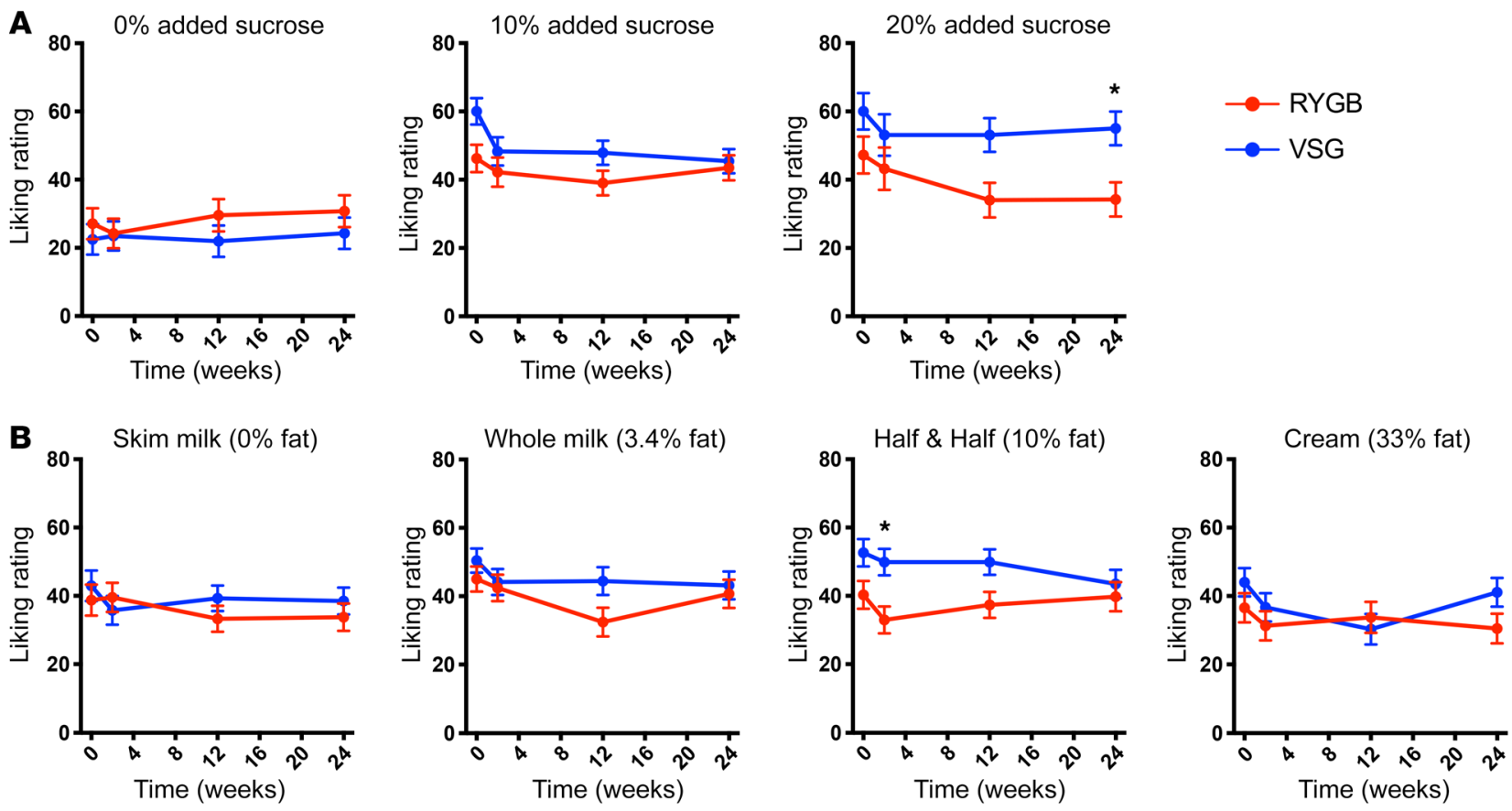

C Cream, $0 \%$ added sucrose

Cream, $0 \%$ added sucrose
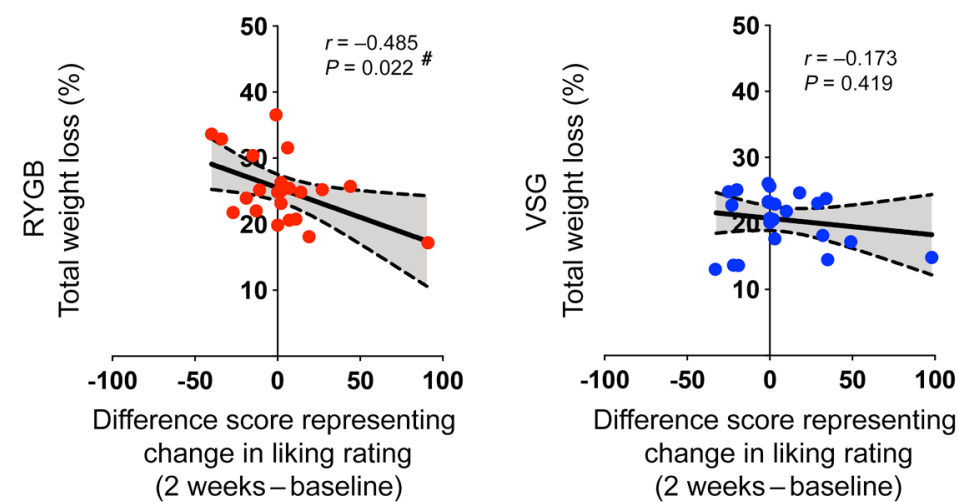

Figure 3. Changes in taste preference following bariatric surgery. (A and B) Mean \pm SEM liking ratings from bariatric patients using the VAS for the 12 mixtures of varying concentrations of sucrose and fat presented in the taste-preference test. (A) Ratings for sucrose content collapsed across all fat contents and (B) ratings for fat content collapsed across all sucrose concentrations before ( 0 weeks) and 2 weeks, 3 months, and 6 months following RYCB (red; $n=21$ ) or VSG (blue; $n=22$ ). Repeated-measures ANOVA identified a main effect of time for $10 \%\left(F_{3,23}=5.430, P=0.002\right)$ and $20 \%\left(F_{3,123}=3.244, P=\right.$ $0.024)$ added sugar and $3.4 \mathrm{~g}$ of fat $\left(F_{3,123}=3.796, P=0.012\right)$. A main group effect was found for $20 \%$ added sucrose $\left(F_{1,41}=6.184, P=0.017\right)$ and $10 \mathrm{~g}$ fat $\left(F_{1,41}\right.$ $=6.992, P=0.012)$. No other main effects or significant interactions were observed $(P \geq 0.071)$. (C) Bivariate correlations of difference scores demonstrating changes in taste-preference ratings for cream with no added sucrose ( 2 weeks after surgery - baseline) in patients who received RYCB (red; $n=22$ ) or VSC (blue; $n=24$ ) and weight-loss metrics at 6 months after surgery. "Significant bivariate correlations. ${ }^{*} P<0.05$.

Changes in liking ratings 2 weeks following RYGB, but not VSG, are associated with 6-month weight loss. Difference scores representing postoperative changes in liking that occurred 2 weeks following surgery before sustained weight loss were calculated to identify any association of short-term taste-preference changes on \%TWL. Following RYGB, decreases in liking for high fat (cream, $0 \%$ added sucrose) correlated with greater \%TWL at 6 months $(P=0.022$; Figure $3 C)$. No association between postoperative changes in taste ratings and \%TWL was observed following VSG.

\section{Preoperative neural (fMRI) associations with weight loss}

Lower preoperative taste-induced BOLD signaling in the VTA is associated with greater 6-month weight loss following RYGB, but not VSG.
Preoperative activation in the following a priori reward regions of interest (ROI) to the high-fat (cream, 0\% added sucrose), high-sugar (skim milk, 20\% added sucrose), and preoperative preferred mixtures did not correlate with 6-month \%TWL: amygdala, insular cortex plus Rolandic operculum, caudate, and putamen. Preoperative responses in the VTA ROI to the high-fat (cream, 0\% added sucrose), high-sugar (skim milk, 20\% added sucrose), and preoperative preferred mixtures were negatively correlated with \%TWL at 6 months following RYGB (high fat: $P=0.006$, high sugar: $P=0.006$, preoperative preferred mixtures: $P=0.019$; Figure 4 and Supplemental Table 3). Participants who exhibited reduced VTA responses before RYGB experienced more weight loss after surgery than those who exhibited greater 


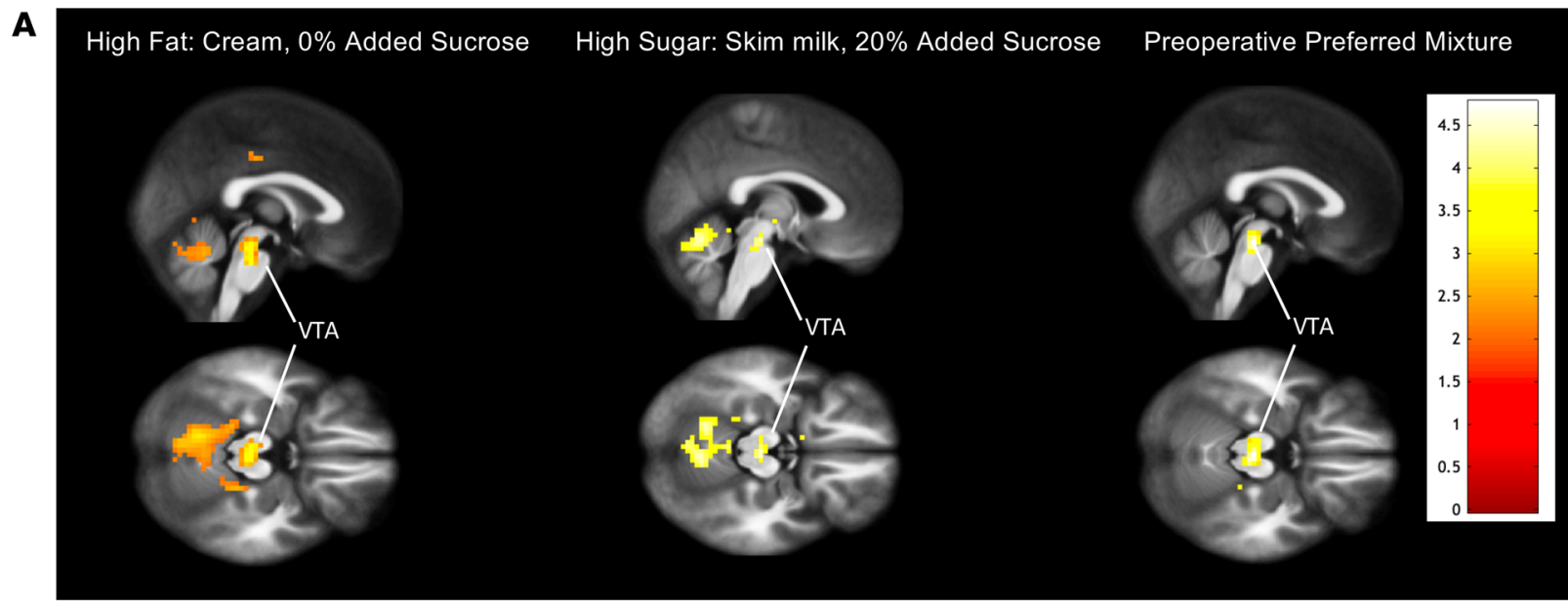

B
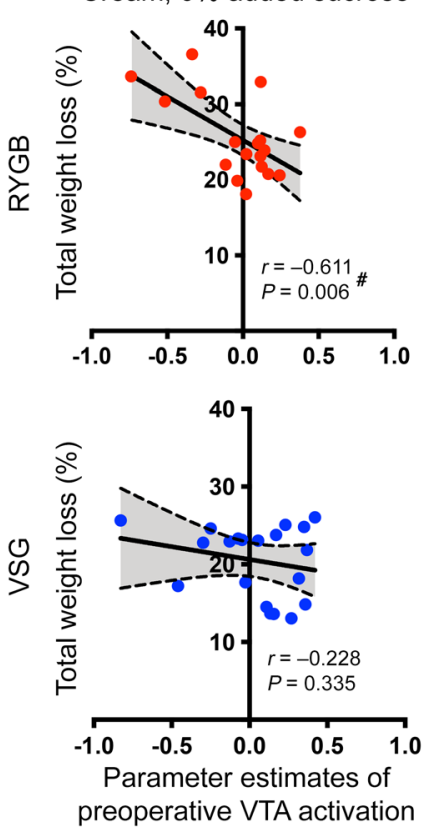

C Skim milk, $20 \%$ added sucrose
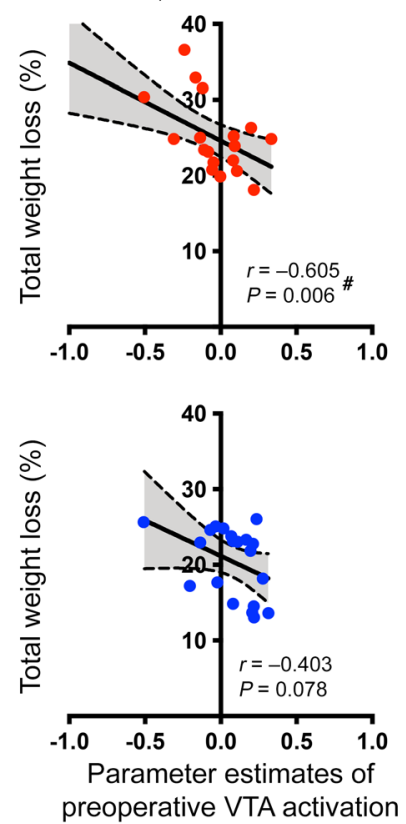

D Preoperative preferred mixture
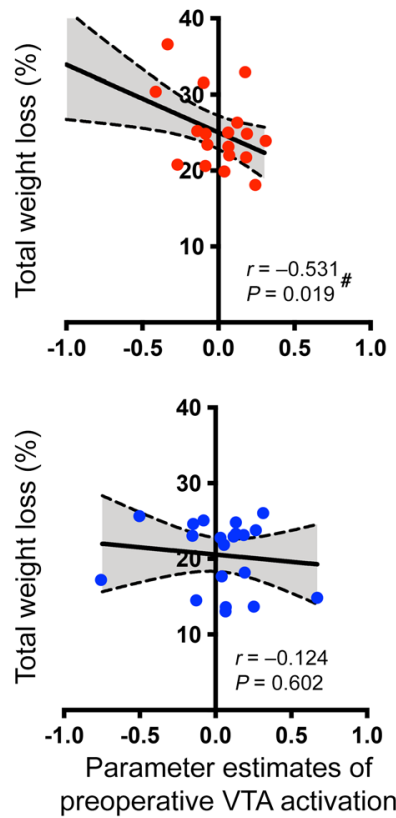

Figure 4. Preoperative activation in the VTA to taste mixtures predicts 6-month weight loss following RYGB. (A) Maps depicting areas within VTA ROI showing negative correlation between baseline activation to the high-fat (cream, $0 \%$ added sucrose), high-sucrose (skim milk, 20\% added sucrose), and preoperative preferred mixtures and weight loss at 6 months in RYCB patients. (B-D) Bivariate correlations demonstrating the relationship between preoperative VTA activation in response to the (B) high-fat, (C) high-sucrose, and (D) most preferred preoperative mixtures and 6-month weight loss following bariatric surgery. Activation in the VTA at baseline to these mixtures presented in the scanner correlated with \% TWL at 6 months following RYCB (red, $n=$ 19), but not VSG (blue, $n=20$ ). "Significant bivariate correlations.

VTA responses before RYGB. In contrast, there was no correlation between preoperative VTA responses and changes in body weight at 6 months following VSG (Figure 4).

\section{Postoperative neural (fMRI) associations with weight loss}

Greater changes in taste-induced BOLD signaling in the VTA 2 weeks following RYGB, but not VSG, is associated with increased 6-month weight loss. Short-term changes in VTA activation in response to the high-fat $(P=0.012)$ and high-sucrose $(P=0.035)$ mixtures, but not the preoperative preferred mixture $(P=0.288)$, were positively associated with \%TWL at 6 months following RYGB (Figure 5 and Supplemental Table 4). Participants who showed greater taste- induced changes in BOLD signaling in the VTA at 2 weeks relative to baseline experienced more weight loss following RYGB than those who exhibited smaller changes. No such relationship was identified in the VSG group (Figure 5).

\section{Discussion}

Our data support the effectiveness of both VSG and RYGB for the treatment of obesity. Currently, VSG is the most common bariatric procedure performed due to technical ease, reduced early postoperative complications (35), and assumed efficacy similar to that of RYGB. We found that RYGB resulted in greater weight loss than VSG at as early as 6 months after surgery (Figure 2). Early postop- 


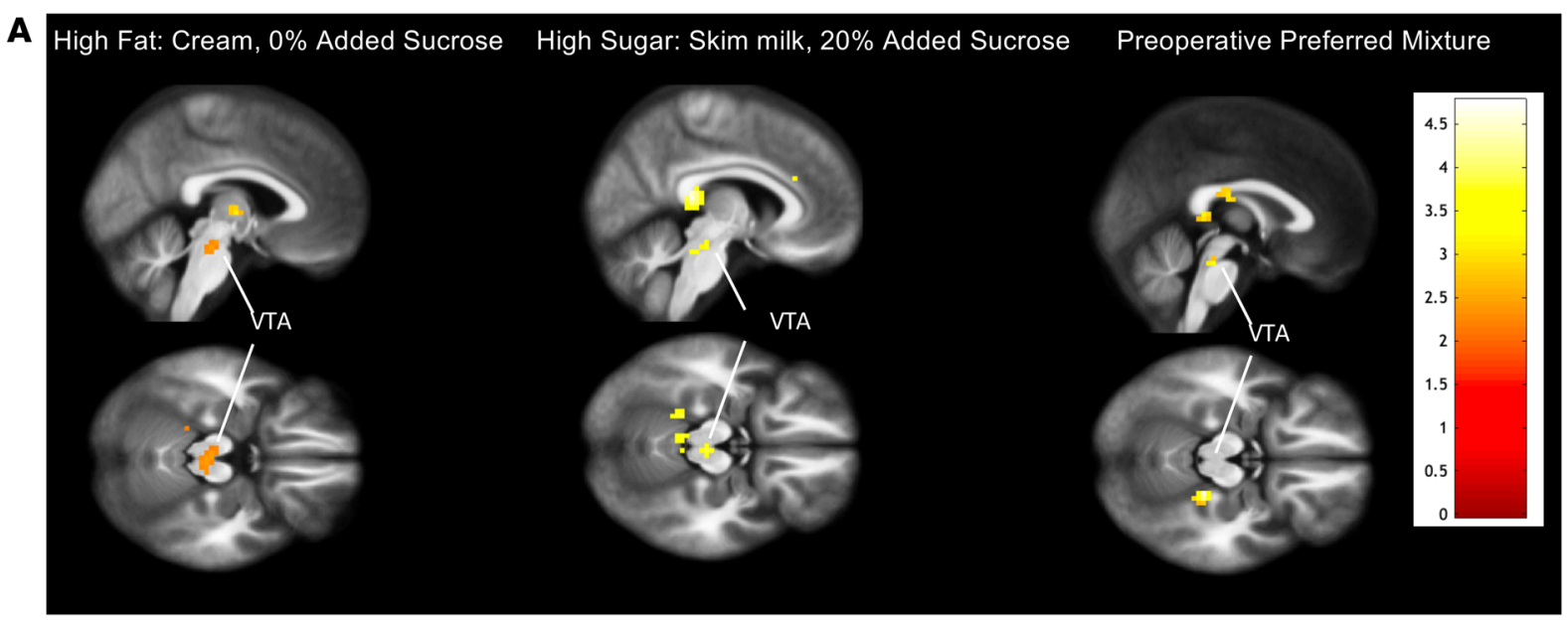

B
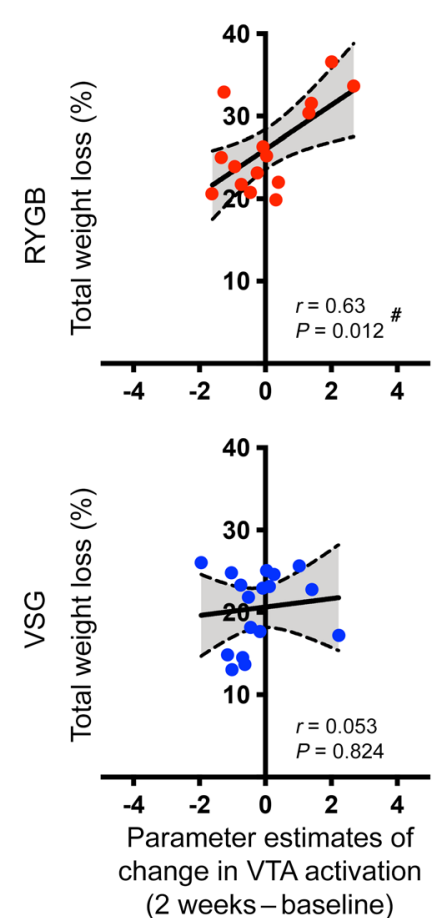

C Skim milk, $20 \%$ added sucrose
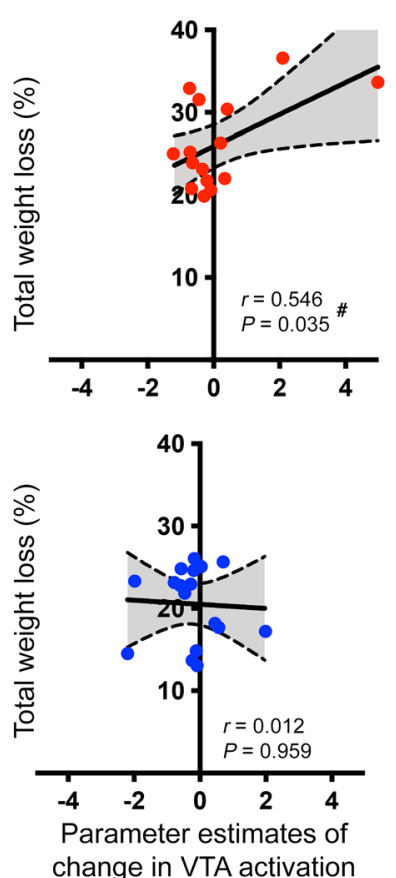

change in VTA activation

(2 weeks-baseline)
D Preoperative preferred mixture
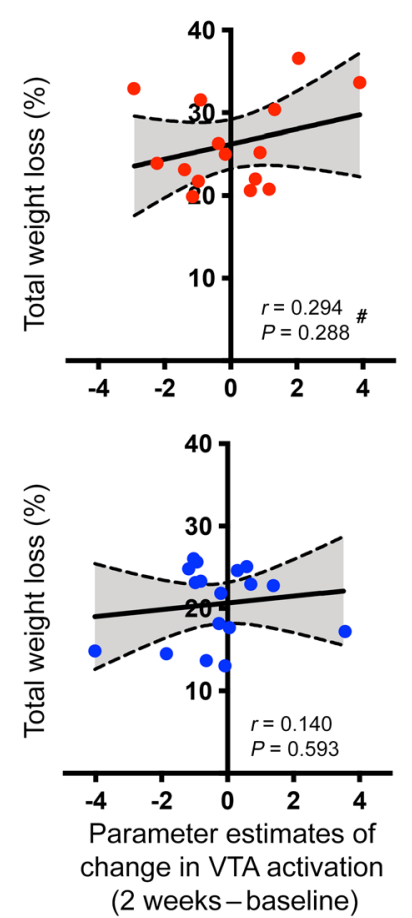

Figure 5. Changes in VTA activation to high-fat and high-sucrose taste mixtures predicts 6-month weight loss following RYGB. (A) Maps depicting areas within VTA ROI showing negative correlation between change in activation to the high-fat (cream, 0\% added sucrose), high-sucrose (skim milk, $20 \%$ added sucrose), and preoperative preferred mixtures from baseline to 2 weeks following surgery and weight loss at 6 months in RYCB patients. (B and D) Bivariate correlations demonstrating the relationship between changes in activation in the VTA to taste mixtures with weight loss at 6 months following surgery. Changes in BOLD signaling in the VTA from baseline to 2 weeks following surgery in response to the (B) high-fat and (C) high-sucrose mixtures correlated with \%TWL at 6 months following RYGB (red, $n=15$ ), but not VSC (blue, $n=17$ ). Changes in VTA activation in response to the most preferred mixture (D) did not correlate with 6-month weight loss. "Significant bivariate correlations.

erative weight loss with both RYGB and VSG is likely driven by a profoundly negative energy balance and catabolic state. Decreased hunger may also influence initial weight loss. We found reductions in subjective fasting hunger ratings at 2 weeks following bariatric surgery, which did not persist at later measurements (Supplemental Figure 2). Changes in hunger may therefore be partially responsible for initial reductions in food intake and subsequent weight loss, but are unlikely to drive the neural and behavioral alterations and weight loss success observed at later time points here.
Preoperative liking ratings of mixtures with added sucrose were positively associated with greater \%TWL in RYGB, but not VSG. Our results suggest that preoperative taste preference could function as a clinical predictor of weight loss success. Specifically, bariatric surgical patients undergoing RYGB who expressed a strong liking (51-100 on a VAS from 0-100) for a sweet-tasting mixture of half-and-half with $10 \%$ added sucrose experienced greater \%TWL than those who rated this sweet mixture less highly (0-50 on the scale). This predictive effect of taste prefer- 
ence did not extend to patients undergoing VSG. VSG recipients, regardless of liking rating, lost an amount of weight equivalent to that of RYGB patients who did not rate the sweet mixture highly (Figure 2). Our results support investigation of a potential tool for precision medicine whereby a clinician could elicit a patient's sweet-mixture ratings during preoperative evaluation and recommend RYGB over VSG for those providing a high rating.

Bariatric surgery, particularly RYGB, resulted in acute decreases in liking for taste mixtures with added sugar and fat, consistent with previous literature (36). Indeed, RYGB eliminated concentration-dependent responses to mixtures with added sucrose, and RYGB patients who demonstrated a decreased preference for fat 2 weeks postoperatively showed significantly more \%TWL at 6 months than those who showed no change or increased taste preference for fat (Figure 3). No such effects were apparent for the VSG group. Together, the above findings suggest that the anatomical and/or metabolic changes associated with RYGB may more effectively "reset" the neural processing of reward stimuli, thereby rescuing the blunted activation of the mesolimbic pathway found in patients with obesity. Further, they suggest that RYGB may be particularly effective in patients with a preference for sweet foods. This is consistent with the hypothesis that RYGB facilitates weight loss by decreasing an initial high liking for energy-dense foods, which in turn may reduce selection and consumption of such foods after surgery (26-30).

Foods that are high in sugar and fat stimulate reward circuits that promote their future consumption, and chronic exposure to such foods may result in blunted responses to those foods in brain reward circuits $(18,19)$. BOLD signaling, while a proxy of neural activation, has been shown to reflect dopaminergic activity in the VTA (37). We used BOLD fMRI to show for what we believe is the first time that responsiveness at this central reward site to palatable, high-energy dense mixtures is associated with weight loss following bariatric surgery. We found that patients who exhibited lower BOLD responses in the VTA to palatable mixtures before RYGB lost more weight 6 months after surgery than RYGB recipients who exhibited higher VTA responses (Figure 4). Preoperative BOLD responses in the VTA to palatable mixtures were not correlated with changes in body weight at 6 months following surgery in patients who underwent VSG. Further, following RYGB only, greater 2-week changes in VTA responses to high-fat and high-sucrose mixtures were associated with greater \%TWL at 6 months (Figure 5). The short-term changes in VTA responsivity within 2 weeks of surgery may reflect a restoration of formerly blunted neural responses that could be partly driven by improved dopaminergic function in individuals with obesity following bariatric surgery, specifically RYGB, and may be responsible for postoperative shifts in dietary choices toward less energy-dense foods (26-30). These results are also consistent with our previous work showing that increases in dopamine receptor availability following bariatric surgery are approximately proportional to the amount of weight loss (16). Similar RYGB-specific BOLD changes in the VTA in response to high-calorie food cues relative to low-calorie food cues despite equivalent weight loss between RYGB and VSG patients have been observed (38). Faulconbridge et al. also found changes in VTA BOLD responses to be positively associated with changes in fasting ghrelin following bariatric surgery, pointing to a possible mechanism of action (38). However, this association was not specific to RYGB; changes in fasting ghrelin after both VSG and RYGB correlated with changes in VTA BOLD responses.

Baseline behavioral or biological predictors of variability in surgery-induced weight loss hold the most clinical value in their potential to guide treatment choice. However, we also explored whether early changes in taste preference could predict treatment response, since early identification of individuals at higher risk for poor weight loss could guide delivery of postoperative interventions. While these findings require replication in a larger sample and longer term follow-up, they suggest that surgery-specific effects on reward function occur early following surgery and may ultimately contribute to procedure-specific differences in weight loss.

Limitations. Patients were not randomly assigned to a surgical intervention. Other than the higher prevalence of GERD, our patient population was well matched between surgical groups (see Table 1). The higher prevalence of GERD in the RYGB group is not surprising, given the increasing evidence of postoperative GERD-related issues following VSG. Patients with marked preoperative GERD symptoms are generally encouraged to consider RYGB. We did not measure adherence to postoperative lifestyle recommendations, such as dietary modification. Future studies should include explicit assessments of dietary intake to ascertain bidirectional relationships between taste-preference changes and eating behaviors following surgery. While our sample size was sufficiently large to detect surgery-induced changes in taste preferences, larger studies in a separate sample are necessary to validate the ability of the behavioral and neural markers we identified here to predict weight loss from RYGB and VSG. We only administered the high-fat with $0 \%$ added sucrose and the skim milk with $20 \%$ added sucrose solutions to all subjects in the scanner. Recent work has demonstrated that combinations of fat and sugar produce supraadditive effects on neural activation (39). The addition of a high-sucrose, high-fat combination may produce more robust neuroimaging results, adding strength to predictions.

Conclusions. This study is the first, to our knowledge, to directly measure reductions in liking for and changes in BOLD signaling in response to sucrose- and fat-containing mixtures and associate these with weight-loss outcomes. Our findings suggest that bariatric surgery may correct brain responses to high-sucrose and high-fat food stimuli within circuits mediating reward function. These results constitute what we believe is the first evidence for a specific neurobiologic mechanism for postoperative changes in taste preference. Such changes in taste preference may affect food intake and weight reduction. We also demonstrate potential predictors of weight loss specific to surgery type. Greater preoperative liking of sugar-sweetened mixtures is associated with greater 6-month weight loss among RYGB patients. Lower preoperative VTA activation and greater 2-week changes in VTA response to high-sugar and high-fat mixtures correlated with improved weight loss with RYGB, but not VSG. Our findings further illuminate the neurobiological correlates of reward and its connection to obesity and weight loss, with practical implications for clinicians involved in the care of preoperative bariatric patients. 


\section{Methods}

Participants. Male and female adults, 18 to 55 years of age with a BMI of 35 or more and approved for bariatric surgery at the Johns Hopkins Center for Bariatric Surgery were recruited for this cohort study (see Figure 1). Participants were ineligible if they met any of the following criteria: (a) had an active DSM-IV axis 1 diagnosis within the past 3 months, with the exception of binge-eating disorder; (b) had a DSM-IV diagnosis of substance-dependent disorder; (c) reported or tested positive for drug use within the past 6 months; (d) had a history of heavy smoking ( $>2$ packs of cigarettes/d); (e) consumed more than 14 alcoholic beverages/wk or 5 or more alcoholic beverages/d; (f) were actively taking psychoactive medications; (g) were pregnant or lactating; (h) were reading below a fifth-grade level; (i) had a history of head injury, central nervous system disorders, neurosurgical procedures, or syncope; (j) were lactose intolerant; (k) received recent (within 3 months) treatment for 2 or more weeks with antidepressants, neuroleptics, sedatives, hypnotic medications, isoniazids, glucocorticoids, psychostimulants, appetite suppressants, opiates, or opiate antagonists; (l) had an inability to undergo MRI (e.g., claustrophobia, metal implant, pacemaker, foreign body); (m) were exposed to an investigational drug within 30 days of the study; or (n) had a history of altered taste acuity.

Initial participant sample size was $n=53$. Originally, 3 of the 5 male participants enrolled in this study were approved and scheduled for RYGB. However, due to intraoperative findings and safety considerations, the surgeon opted for VSG rather than the planned RYGB. To eliminate sex as a confounding variable, the 5 males were not included in the analyses, and therefore only data from females within the VSG group were included. Final participant sample size for each group was as follows: RYGB, $n=23$; VSG, $n=25$ (total $n=48$ ). All study participants were female.

Study measures were collected across 4 time points: 2 weeks \pm 14 days before surgery, 2 weeks \pm 14 days after surgery, 3 months \pm 30 days after surgery, and 6 months \pm 30 days after surgery. All participants were asked to refrain from eating or drinking, with the exception of water, at least 4 hours before each study assessment. Participant weight and height were measured using a high-capacity digital scale and stadiometer, respectively. If a participant was lost to follow-up, her body weight was gleaned from the Johns Hopkins Hospital electronic medical record system (EPIC) if available. Participants then underwent a modified version of the taste-preference test employed by Drewnowski et al. (40).

Taste-preference test. Twelve taste mixtures of varying fat (milk) and sugar (sucrose) content were presented in randomized order to each participant in clear 30-cc disposable plastic cups and given an arbitrary number-letter label for patient scoring purposes. These mixtures were skim milk, whole milk, half-and-half, and cream prepared with either $0 \%, 10 \%$, or $20 \%$ added sugar. Each participant sampled each taste mixture, swished it in her mouth, and rated her liking of the stimulus on a 100-mm hedonic VAS anchored by "not at all" to "very much" on the left and right, respectively, before expectoration. A water rinse was presented after expectoration of each taste sample. Ratings on the VAS scale were recorded using Qualtrics survey software. At the conclusion of each taste test, patients were asked to confirm the mixture that they most preferred. Each participant also selected 1 of 4 solutions composed of varying concentrations of sodium bicarbonate and potassium chloride that were designed to mimic the tasteless nature of saliva (41) (for application in the fMRI paradigm). Before the taste assessments, participants rated their levels of hunger on a similar 100-mm VAS anchored by "not at all" to "very much."

fMRI paradigm. The taste-preference test always preceded the fMRI paradigm. All participants underwent standard MRI screening procedures before MRI scanning. An MRI-compatible gustometer delivered taste mixtures in a block design and in pseudorandomized order to the participant's mouth during scanning for measurement of real-time, taste-induced BOLD responses (paradigm modeled after Veldhuizen et al.; ref. 42). Patients were instructed to focus on how much they liked or disliked the taste of the solution during oral infusion. BOLD responses to the high-fat (cream, $0 \%$ added sugar) and high-sugar (skim milk, 20\% added sugar) mixtures presented in the taste-preference array described above were assessed at 2 time points: 2 weeks \pm 14 days before surgery and 2 weeks \pm 14 days after surgery. For the preoperative/baseline scan, the following taste mixtures were delivered at a rate of $0.4 \mathrm{~mL} / 4 \mathrm{~s}$ delivered across 66 trials in 4 runs: a high-fat mixture (cream, $0 \%$ added sugar), a high-sugar mixture (skim, 20\% added sugar), and the participant's most preferred mixture and the "tasteless" solution selected during the preoperative/ baseline taste test. A 4-second water rinse was delivered following each taste solution presentation. Postsurgical/follow-up scans were conducted identically to the preoperative/baseline scans, with the following adjustments to stimulus delivery: (a) the participant's most preferred stimulus at the postoperative/follow-up taste test was added to the fMRI stimulus-delivery array, thus increasing the trial number to 77 trials to facilitate the addition of the participant's most preferred stimulus assessed at the respective postsurgical/follow-up taste test; and (b) the tasteless solution selected at the respective postsurgical/ follow-up taste test was substituted for the previously delivered preoperative tasteless solution. Six programmable syringe pumps (NE-1000, New Era Pump Systems Inc.) delivered the mixtures to the participant from 60-mL syringes via Tygon tubing (McMaster, catalog 5103K32; one-eighth-inch inside diameter) to a manifold affixed to the sliding table of the fMRI scanner (3.0 Tesla Phillips HealthCare). Stimulus delivery was controlled using MATLAB (R2017b; MathWorks Inc.). A multiple-element 32-channel receiver head coil was used to acquire BOLD-weighted MRI data using single-shot SENSE-EPI $(\mathrm{TR} / \mathrm{TE}=$ $2000 / 30 \mathrm{~ms}$; flip angle $=70^{\circ} ; 3 \times 3 \times 3 \mathrm{~mm}^{3}$ resolution; acquisition matrix, $64 \times 52$; 3-mm-thick slices; field of view [FOV], $192 \times 156 \times$ $117 \mathrm{~mm}$; acquisition of 39 contiguous slices). High-resolution anatomical images were acquired using a T1-weighted 3D magnetization-prepared rapid acquisition gradient-echo (MPRAGE) sequence $(\mathrm{TR} / \mathrm{TE}=$ 8.0/3.70 ms; flip angle $=8^{\circ} ; 1 \times 1 \times 1 \mathrm{~mm}^{3}$ resolution; acquisition matrix, $212 \times 172$; 1-mm-thick slices; FOV, $212 \times 172 \times 150 ; 150$ slices). Slices were acquired in ascending mode. A clinical radiologist reviewed all scans for identification of abnormalities in brain structure.

Statistics. Behavioral and anthropometric data were analyzed using SPSS Statistics software, and fMRI data were analyzed using SPM12 (Wellcome Department of Cognitive Neurology) with MATLAB software. The $\alpha$ level was established at 0.05 , and analyses were controlled for multiple comparisons when applicable. The Greenhouse-Geisser correction was used to produce a more valid critical $F$ value when sphericity was violated. One-way ANOVA was conducted on age, preoperative/baseline BMI, and preoperative/baseline body weight; and $\chi^{2}$ analyses were conducted on race and obesity comorbidities (i.e., type 2 diabetes, hypertension, hyperlipidemia, sleep apnea, GERD), with surgery group as the between-subjects factor to assess 
differences between groups in patient demographics. A repeated-measures (surgery group $\times$ time) ANOVA and follow-up paired-samples $t$ tests were performed on hunger ratings to assess changes between and within surgical groups across time (RYGB: $n=20$, VSG: $n=20$ ).

Effectiveness of bariatric procedures was assessed by repeated-measure ANOVAs (surgery group $\times$ time) on change in BMI, \%TWL, and \%EWL. The following formulas were used to calculate \%TWL and $\% \mathrm{EWL}: \% \mathrm{TWL}=([$ preoperative or baseline weight $]-$ [postoperative weight] $) /($ preoperative or baseline weight] $) \times 100 ; \% \mathrm{EWL}=([$ preoperative or baseline weight] - [postoperative weight])/([preoperative or baseline weight] - [ideal weight] $\times 100$, with ideal weight being defined as the weight equivalent to a BMI of $25 \mathrm{~kg} / \mathrm{m}^{2}$. Linear interpolation was used to calculate body-weight values for 2 VSG patients - one who missed a 2-week and another who missed a 3-month visit because body-weight data were not available in EPIC, and $t$ tests were run to compare changes in body weight across time between surgical groups when a significant main effect or interaction was identified. Sample size for these analyses were as follows: RYGB, $n=23$; VSG, $n=25$. Bivariate correlations were also conducted on \%TWL, change in $\mathrm{BMI}$, and \%EWL at 6 months following surgery with preoperative liking ratings of each taste mixture. Patients who completed the baseline taste visits and whose 6-month weights were available were included in these analyses (RYGB, $n=23$; VSG, $n=25$ ).

ICCs were conducted on preoperative taste ratings to determine the reliability of ratings across each sugar and fat group. To assess changes in taste preferences between surgical groups across time, repeated-measures ANOVAs (surgery group $\times$ time) were conducted on ratings of the mixtures collapsed across each sucrose $(0 \%, 10 \%$, $20 \%$ ) and fat (skim, whole, half-and-half, cream) concentration. Repeated-measures ANOVAs (surgery group $\times$ concentration) were run on ratings of the mixtures collapsed across each sucrose $(0 \%$, $10 \%, 20 \%)$ and fat (skim, whole, half-and-half, cream) concentration at each time point to determine the effect of bariatric surgery on concentration-dependent responding. One-way repeated-measures ANOVAs were then conducted to determine the effects of bariatric surgery on concentration-dependent responding within each group. One-way repeated-measures ANOVAs and $t$ tests were run to compare changes in VAS ratings when a significant main effect or interaction was identified. Only patients who completed all 4 taste visits were included in these analyses (RYGB, $n=21$; VSG, $n=22$ ).

Difference scores were then used to determine the extent to which taste changes induced by bariatric surgery predicted 6-month weight loss success (\%TWL). Difference scores were calculated by subtracting the 2-week postsurgical rating of each taste mixture from the preoperative/baseline rating, where positive values represent increases in preference and negative values represent decreases in preference for a mixture following surgery. Bivariate correlations were conducted on \%TWL from surgery with difference scores at 2 weeks for each taste mixture. Patients who completed the baseline and 2-week postoperative taste visits and whose 6-month weights were available were included in these analyses (RYGB, $n=22$; VSG, $n=24$ ).

Preoperative/baseline hedonic ratings were explored as potential preoperative predictors of optimal weight-loss success. Bariatric patients were subdivided into 4 groups (surgery group $\times$ liking group) based on their preoperative liking ratings of each taste mixture. The 2 liking groups included patients who rated the respective mixtures on the VAS with a 51-100 before surgery (preferrers) and those who rated the mixture with a 0-50 before surgery (nonpreferrers). Repeated-measures ANOVAs (surgery/taste group $\times$ time) were then run on \%TWL. Post hoc $t$ tests were run to compare changes in body weight across time between surgical/taste groups when a significant main effect or interaction was identified.

Functional images were slice time corrected using the median slice as the reference slice and realigned to the mean of the images after the initial realignment. Functional images were then coregistered to the individual's anatomical T1 image. For 1 participant, the preoperative functional scans, but not structural scans, were acquired. In this case, the patient's 2-week postoperative structural scan was used for coregistration. Images were segmented and normalized to the Montreal Neurological Institute template (43) applying forward deformation fields, resulting in a voxel size of $3 \mathrm{~mm}^{3}$ for functional images and a voxel size of $1 \mathrm{~mm}^{3}$ for structural images. The functional time-series data were detrended, removing any linear global signal component from each voxel's time course. An 8-mm full-width, half-maximum isotropic Gaussian kernel was then used to smooth the images. The artifact detection tools (ART) toolbox for MATLAB was used to detect global mean and motion outliers in the functional data (Gabrieli Laboratory, Massachusetts Institute of Technology, Cambridge, Massachusetts, USA). For first-level analyses, motion parameters were included as regressors in the design matrix and a high-pass filter (256 seconds) was included in the fMRI model specifications to remove low-frequency noise and signal drifts. Additionally, functional image volumes in which the $z$ normalized global brain activation exceeded 3 SDs from the mean of the run or showed more than $1 \mathrm{~mm}$ of composite scan-to-scan movement were flagged as outliers and deweighted during SPM estimation. Tastant-specific effects (e.g., high-fat solution, high-sucrose solution) were estimated using the general linear model. This model was then applied to the canonical hemodynamic response function and its temporal and dispersion derivatives. Events of interest in the preoperative/baseline and postoperative/follow-up scans were defined as blocks of 10-minute, 20-second duration and 12-minute, 50-second duration, respectively, from taste onset. High fat-tasteless, high sugar-tasteless, and preoperative preference-tasteless were entered as contrasts of interest into second-level analyses. Contrasts were equally weighted. For second-level analyses, within- and between-group comparisons were performed using random effects models and 2-sample $t$ tests. For within-subject analyses, the measurements were assumed to be dependent. and for between-subject analyses, the measurements were assumed to be independent.

Anatomical a priori ROI implicated in the neural processing of taste (see refs. 44-47 for review) and reward consisted of the bilateral amygdala, insular cortex, Rolandic operculum, caudate, putamen, and VTA. ROI analyses were performed using the WFU PickAtlas toolbox (WFU_PickAtlas_3.0.5b; refs. 32-34). Masks for the bilateral amygdala, insular cortex and Rolandic operculum (combined), caudate, and putamen were defined using automated anatomical labeling from the WFU PickAtlas. The mask for the VTA was adapted from the highresolution in vivo probabilistic atlas from Pauli et al. (48). Supplemental Figure 4 depicts masks used for ROI analyses. Small-volume correction through SPM was implemented and centered on each of the ROIs. For displaying the results, we used T-contrast maps thresholded at a peak level of $P<0.005$. For reporting the results, activation within each ROI was considered significant at a peak level of $P<0.05$, family-wise error (FWE) corrected. Correlations were conducted 
using multiple regression in SPM to test associations between baseline BOLD activation and changes in weight-loss metrics for each surgery group separately (RYGB, $n=19$; VSG, $n=20$ ). When a significant ROI $\times$ weight-loss correlation was observed, associations between changes in BOLD activation within the ROI at 2 weeks following surgery from preoperative/baseline were assessed for each surgery group separately (RYGB, $n=15$; VSG, $n=17$ ). Results were considered significant at a cluster level of $P<0.05$, FWE corrected for multiple comparisons. For the significance results, parameter estimates from the baseline BOLD activation within the ROI were extracted and plotted against \%TWL.

Study approval. The protocol was approved by the Johns Hopkins IRB. Each participant provided oral or written consent at the initial visit.

\section{Author contributions}

KES was responsible for the principal concept and design of this study. All IRB regulatory preparation and approval and documentation, including preparing all standard operating procedures and maintenance of subject charts, IRB updates/changes in research, and continuing reviews, were the sole responsibility of KES. As PI, KES also made it her responsibility to identify and contact subjects, engage in recruitment and successful retention of participants, complete the full consent process, schedule study visits, complete documentation and compensation of participants, and conduct subject visits; run the experiments, including setup and breaking down of equipment (cleaning and maintenance of the gustometer), collection and cleaning of data, participation in data analysis, contribution of bariatric surgical clinical expertise; and prepare the manuscript, including writing, reviewing, and editing. All authors met for research meetings on a weekly basis throughout the study period. The following were specific author contributions of our team: KES, THM, SC, and VK conceived and designed the experiments. KES, $\mathrm{CH}$, KRS, and AP performed the experiments. KRS, MGV, and AP analyzed the data. KES, $\mathrm{CH}$, and
KRS were responsible for IRB regulatory work and enrollment of participants. KRS, AP, MGV, VK, CH, THM, SC, and KES wrote and revised the manuscript.

\section{Acknowledgments}

The authors, especially the principal investigator, KES, would like to thank the following: Eric Stice for his encouragement, mentorship, guidance and valuable discussions of neuroimaging, taste, and obesity; your passion for the topic inspired this project; Susan Oh, Director, Research Nutrition Program, School of Medicine, Institute of Clinical Translational Research, Johns Hopkins University School of Medicine, and her team of research nutritionists and technicians, who prepared the taste mixtures for over 300 visits; Kevin Colbert and Matt Petney of the Johns Hopkins Center for Bioengineering Innovation and Design for solving the problem of how to pump half-and-half, cream, and sugar through 50 feet of tubing in the gustometer; James Pekar, Terri Brawner, Kathleen Kahl, Ivana Kusevic, Kennedy Krieger, and the Kirby Imaging Center for all of your help, guidance, support, patience, and teaching as we successfully ran each participant's taste paradigm; and, most importantly, our patients, who volunteered their time to participate in this project. Without your generous contributions, none of this work could be accomplished. Thank you for helping our team shed light on the neurobiological correlates of taste preference and their connection to obesity and weight loss. This project was supported by NIH grant K23DK100559 (to KES), with additional support from grants from the Johns Hopkins Science of Learning Institute (to KES and SC) and Dalio Philanthropies (to THM).

Address correspondence to: Kimberley E. Steele, Johns Hopkins University School of Medicine, Attention: Gregory Paul Prokopowicz, 601 N. Caroline Street, JHOC 7157, Baltimore, Maryland 21287, USA. Phone: 410.955.5528; Email: Steele.KimMD@outlook.com.
1. Adams TD, et al. Health benefits of gastric bypass surgery after 6 years. JAMA. 2012;308(11):1122-1131.

2. Sjöström L, et al. Bariatric surgery and long-term cardiovascular events. JAMA. 2012;307(1):56-65.

3. Schauer PR, et al. Bariatric surgery versus intensive medical therapy in obese patients with diabetes. N Engl J Med. 2012;366(17):1567-1576.

4. Stefater MA, Wilson-Pérez HE, Chambers AP, Sandoval DA, Seeley RJ. All bariatric surgeries are not created equal: insights from mechanistic comparisons. Endocr Rev. 2012;33(4):595-622.

5. Akkary E, et al. The paradox of the pouch: prompt emptying predicts improved weight loss after laparoscopic Roux-Y gastric bypass. Surg Endosc. 2009;23(4):790-794.

6. Dirksen C, et al. Fast pouch emptying, delayed small intestinal transit, and exaggerated gut hormone responses after Roux-en-Y gastric bypass. Neurogastroenterol Motil. 2013;25(4):346-e255.

7. le Roux CW, et al. Gut hormones as mediators of appetite and weight loss after Roux-en-Y gastric bypass. Ann Surg. 2007;246(5):780-785.

8. Peterli R, et al. Metabolic and hormonal changes after laparoscopic Roux-en-Y gastric bypass and sleeve gastrectomy: a randomized, prospective trial. Obes Surg. 2012;22(5):740-748.

9. Borg CM, le Roux CW, Ghatei MA, Bloom SR, Patel AG, Aylwin SJ. Progressive rise in gut hormone levels after Roux-en-Y gastric bypass suggests gut adaptation and explains altered satiety. Br J Surg. 2006;93(2):210-215.

10. Yan W, et al. Effects of meal size on the release of GLP-1 and PYY after Roux-en-Y gastric bypass surgery in obese subjects with or without type 2 diabetes. Obes Surg. 2014;24(11):1969-1974.

11. Jacobsen $\mathrm{SH}$, et al. Changes in gastrointestinal hormone responses, insulin sensitivity, and beta-cell function within 2 weeks after gastric bypass in non-diabetic subjects. Obes Surg. 2012;22(7):1084-1096.

12. Sánchez-Alcoholado L, Gutiérrez-Repiso C, Gómez-Pérez AM, García-Fuentes E, Tinahones FJ, Moreno-Indias I. Gut microbiota adaptation after weight loss by Roux-en-Y gastric bypass or sleeve gastrectomy bariatric surgeries. Surg Obes Relat Dis. 2019;15(11):1888-1895.

13. Brutman JN, Sirohi S, Davis JF. Recent advances in the neurobiology of altered motivation following bariatric surgery. Curr Psychiatry Rep. 2019;21(11):117.

14. Berridge KC. 'Liking' and 'wanting' food rewards: brain substrates and roles in eating disorders. Physiol Behav. 2009;97(5):537-550.

15. Wang JL, Yang Q, Hajnal A, Rogers AM. A pilot functional MRI study in Roux-en-Y gastric bypass patients to study alteration in taste functions after surgery. Surg Endosc. 2016;30(3):892-898.

16. Steele KE, et al. Alterations of central dopamine receptors before and after gastric bypass surgery. Obes Surg. 2010;20(3):369-374.

17. Dunn JP, et al. Decreased dopamine type 2 receptor availability after bariatric surgery: preliminary findings. Brain Res. 2010;1350:123-130.

18. Ochner CN, et al. Relation between changes in neural responsivity and reductions in desire to eat high-calorie foods following gastric bypass surgery. Neuroscience. 2012;209:128-135.

19. Ochner CN, et al. Selective reduction in neural responses to high calorie foods following gastric bypass surgery. Ann Surg. 2011;253(3):502-507.

20. Zoon HFA, et al. Altered neural responsivity to food cues in relation to food preferences, but not appetite-related hormone concentrations after RYGB-surgery. Behav Brain Res. 2018;353:194-202.

21. Burge JC, Schaumburg JZ, Choban PS, DiSilvestro RA, Flancbaum L. Changes in patients' taste acuity after Roux-en-Y gastric bypass 
for clinically severe obesity. J Am Diet Assoc. 1995;95(6):666-670.

22. Bueter $M$, et al. Alterations of sucrose preference after Roux-en-Y gastric bypass. Physiol Behav . 2011;104(5):709-721.

23. Altun $\mathrm{H}$, et al. Improved gustatory sensitivity in morbidly obese patients after laparoscopic sleeve gastrectomy. Ann Otol Rhinol Laryngol. 2016;125(7):536-540.

24. Nance K, Eagon JC, Klein S, Pepino MY. Effects of sleeve gastrectomy vs. Roux-en-Y gastric bypass on eating behavior and sweet taste perception in subjects with obesity. Nutrients. 2017;10(1):E18.

25. Rodin J, Moskowitz HR, Bray GA. Relationship between obesity, weight loss, and taste responsiveness. Physiol Behav. 1976;17(4):591-597.

26. Thomas JR, Gizis F, Marcus E. Food selections of Roux-en-Y gastric bypass patients up to 2.5 years postsurgery. JAm Diet Assoc. 2010;110(4):608-612.

27. Ernst B, Thurnheer M, Wilms B, Schultes B. Differential changes in dietary habits after gastric bypass versus gastric banding operations. Obes Surg. 2009;19(3):274-280.

28. Brolin RE, Robertson LB, Kenler HA, Cody RP. Weight loss and dietary intake after vertical banded gastroplasty and Roux-en-Y gastric bypass. Ann Surg. 1994;220(6):782-790.

29. Kenler HA, Brolin RE, Cody RP. Changes in eating behavior after horizontal gastroplasty and Roux-en-Y gastric bypass. Am J Clin Nutr. 1990;52(1):87-92.

30. Kruseman M, Leimgruber A, Zumbach F, Golay A. Dietary, weight, and psychological changes among patients with obesity, 8 years after gastric bypass. J Am Diet Assoc. 2010;110(4):527-534.

31. Trostler N, Mann A, Zilberbush N, Avinoach E, Charuzi I. Weight loss and food intake 18 months following vertical banded gastroplasty or gastric bypass for severe obesity. Obes Surg. 1995;5(1):39-51.

32. Wardé-Kamar J, Rogers M, Flancbaum L, Laferrère $\mathrm{B}$. Calorie intake and meal patterns up to 4 years after Roux-en-Y gastric bypass surgery. Obes Surg. 2004;14(8):1070-1079.

33. Nielsen MS, et al. Roux-En-Y gastric bypass and sleeve gastrectomy does not affect food preferences when assessed by an ad libitum buffet meal. Obes Surg. 2017;27(10):2599-2605.

34. Coluzzi I, et al. Food intake and changes in eating behavior after laparoscopic sleeve gastrectomy. Obes Surg. 2016;26(9):2059-2067.

35. Osland E, Yunus RM, Khan S, Alodat T, Memon B, Memon MA. Postoperative early major and minor complications in laparoscopic vertical sleeve gastrectomy (LVSG) versus laparoscopic Roux-en-Y gastric bypass (LRYGB) procedures: a meta-analysis and systematic review. Obes Surg. 2016;26(10):2273-2284.

36. Behary P, Miras AD. Food preferences and underlying mechanisms after bariatric surgery. Proc Nutr Soc. 2015;74(4):419-425.

37. D’Ardenne K, McClure SM, Nystrom LE, Cohen JD. BOLD responses reflecting dopaminergic signals in the human ventral tegmental area. Science. 2008;319(5867):1264-1267.

38. Faulconbridge LF, et al. Changes in neural responsivity to highly palatable foods following Roux-en-Y gastric bypass, sleeve gastrectomy, or weight stability: an fMRI study. Obesity (Silver Spring). 2016;24(5):1054-1060.

39. DiFeliceantonio AG, et al. Supra-additive effects of combining fat and carbohydrate on food reward. Cell Metab. 2018;28(1):33-44.e3.

40. Drewnowski A, Halmi KA, Pierce B, Gibbs J, Smith GP. Taste and eating disorders. Am JClin Nutr. 1987;46(3):442-450.

41. O’Doherty J, Rolls ET, Francis S, Bowtell R, McGlone F. Representation of pleasant and aversive taste in the human brain. J Neurophysiol. 2001;85(3):1315-1321.

42. Veldhuizen MG, Bender G, Constable RT, Small DM. Trying to detect taste in a tasteless solution: modulation of early gustatory cortex by attention to taste. Chem Senses. 2007;32(6):569-581.

43. Mazziotta JC, et al. Four-dimensional probabilistic atlas of the human brain. J Am Med Inform Assoc (JAMIA). 2001;8(5):401-430.

44. Veldhuizen MG, Albrecht J, Zelano C, Boesveldt S, Breslin P, Lundström JN. Identification of human gustatory cortex by activation likelihood estimation. Hum Brain Mapp. 2011;32(12):2256-2266.

45. Small DM, Prescott J. Odor/taste integration and the perception of flavor. Exp Brain Res. 2005;166(3-4):345-357.

46. Rolls ET. Taste, olfactory and food texture reward processing in the brain and the control of appetite. Proc Nutr Soc. 2012;71(4):488-501.

47. Small DM. Flavor is in the brain. Physiol Behav. 2012;107(4):540-552.

48. Pauli WM, Nili AN, Tyszka JM. A high-resolution probabilistic in vivo atlas of human subcortical brain nuclei. Sci Data. 2018;5:180063. 